\title{
Hyperon studies at BESIII
}

\section{Andrzej Kupsc* for the BESIII Collaboration}

Uppsala University

E-mail: Andrzej.Kupsc@physics.uu.se

Recent results on $e^{+} e^{-} \rightarrow \Lambda \bar{\Lambda}$ from BESIII Collaboration are reported: (i) for the process at $J / \psi$ and (ii) for the non-resonant production.

(i) Using a data sample of $1.31 \times 10^{9} \mathrm{~J} / \psi$ events we report the first observation of spin polarization of $\Lambda$ and $\bar{\Lambda}$ hyperons. The phase between the helicity flip and helicity non-flip amplitudes of $\Delta \Phi=(42.4 \pm 0.6 \pm 0.5)^{\circ}$ is determined. The decay asymmetries for $\Lambda \rightarrow p \pi^{-}$ $\left(\alpha_{-}\right), \bar{\Lambda} \rightarrow \bar{p} \pi^{+}\left(\alpha_{+}\right)$and $\bar{\Lambda} \rightarrow \bar{n} \pi^{0}\left(\bar{\alpha}_{0}\right)$ are measured to be $\alpha_{-}=0.750 \pm 0.009 \pm 0.004$, $\alpha_{+}=-0.758 \pm 0.010 \pm 0.007$ and $\bar{\alpha}_{0}=-0.692 \pm 0.016 \pm 0.006$, respectively. The obtained value of $\alpha_{-}$is higher by $(17 \pm 3) \%$ than the world average of $0.642 \pm 0.013$ used for all $\Lambda$ polarization measurements since 1978 . The $C P$ asymmetry $A_{C P}=\left(\alpha_{-}+\alpha_{+}\right) /\left(\alpha_{-}-\alpha_{+}\right)$of $-0.006 \pm 0.012 \pm 0.007$ and the ratio $\bar{\alpha}_{0} / \alpha_{+}=0.913 \pm 0.028 \pm 0.012$ are also measured.

(ii) Using $66.9 \mathrm{pb}^{-1}$ collected at $\sqrt{s}=2.396 \mathrm{GeV} \Lambda$ form factors are determined. This is the first complete determination of the time-like elastic form factors $G_{M}$ and $G_{E}$ for any baryon: the ratio $R=\left|G_{E} / G_{M}\right|$ and the relative phase $\Delta \Phi=\arg \left(G_{E} / G_{M}\right)$. Using the decay asymmetry parameters from analysis $(i)$ as input, the obtained values are $R=0.96 \pm 0.14 \pm 0.02$ and $\Delta \Phi=(37 \pm 12 \pm 6)^{\circ}$, respectively. The normalization of the form factors is set by the determined Born cross section cross section of $\sigma_{\text {Born }}=119.0 \pm 5.3 \pm 5.1 \mathrm{pb}$.

In a separate analysis a surprisingly large Born cross section of $305 \pm 45_{-36}^{+66} \mathrm{pb}$ is measured at $\sqrt{s}=2.2324 \mathrm{GeV}$, which is $1.0 \mathrm{MeV}$ above the threshold.

The 39th International Conference on High Energy Physics (ICHEP2018)

4-11 July, 2018

Seoul, Korea

${ }^{*}$ Speaker. 


\section{The $e^{+} e^{-} \rightarrow \Lambda \bar{\Lambda}$ process at the $J / \psi$ resonance $([1,2])$}

The well-defined and simple initial state makes baryon-antibaryon pair production at an electronpositron collider an ideal system to test fundamental symmetries in the baryon sector, in particular when the probability of the process is enhanced by a resonance such as the $J / \psi$ [3]. The spin orientations of the baryon and antibaryon are correlated and, for spin one-half baryons, the pair is produced either with the same or opposite helicities. The transition amplitudes to the respective spin states can acquire a relative phase, $\Delta \Phi$ due to the strong interaction in the final state, leading to a time-reversal-odd observable: a transverse spin polarization of the baryons $[4,5]$. This effect has no prediction and was previously neglected for the baryon pairs from $J / \psi$ decays [6].

The polarization of the $\Lambda$ hyperons, can be determined using the angular distribution of the daughter particles. For example, for the $\Lambda \rightarrow p \pi^{-}$decay with the $\Lambda$ hyperon polarization given by the $\mathbf{P}_{\Lambda}$ vector, the angular distribution of the daughter protons is $\frac{1}{4 \pi}\left(1+\alpha_{-} \mathbf{P}_{\Lambda} \cdot \hat{\mathbf{n}}\right)$, where $\hat{\mathbf{n}}$ is the unit vector along the proton momentum in the $\Lambda$ rest frame and $\alpha_{-}$is the asymmetry parameter of the decay [7]. The corresponding parameters $\alpha_{+}$for $\bar{\Lambda} \rightarrow \bar{p} \pi^{+}, \alpha_{0}$ for $\Lambda \rightarrow n \pi^{0}$, and $\bar{\alpha}_{0}$ for $\bar{\Lambda} \rightarrow \bar{n} \pi^{0}$ are defined in the same way [8]. The joint angular distribution of $J / \psi \rightarrow \Lambda \bar{\Lambda}(\Lambda \rightarrow f$ and $\bar{\Lambda} \rightarrow \bar{f}, f=p \pi^{-}$or $n \pi^{0}$ ) depends on the $\Lambda$ and $\bar{\Lambda}$ polarization and spin correlation of the $\Lambda \bar{\Lambda}$ pair via the $\alpha_{\psi}$ and $\Delta \Phi$ parameters. In particular, the joint angular distribution of the decay chain $J / \psi \rightarrow\left(\Lambda \rightarrow p \pi^{-}\right)\left(\bar{\Lambda} \rightarrow \bar{p} \pi^{+}\right)$can be expressed as [6]:

$\mathscr{W}\left(\boldsymbol{\xi} ; \alpha_{\psi}, \Delta \Phi, \alpha_{-}, \alpha_{+}\right)=1+\alpha_{\psi} \cos ^{2} \theta$

$+\alpha_{-} \alpha_{+}\left\{\sin ^{2} \theta\left(n_{1, x} n_{2, x}-\alpha_{\psi} n_{1, y} n_{2, y}\right)+\left(\cos ^{2} \theta+\alpha_{\psi}\right) n_{1, z} n_{2, z}+\sqrt{1-\alpha_{\psi}^{2}} \cos \Delta \Phi \sin 2 \theta \frac{n_{1, x} n_{2, z}+n_{1, z} n_{2, x}}{2}\right\}$

$+\sqrt{1-\alpha_{\psi}^{2}} \sin (\Delta \Phi) \sin \theta \cos \theta\left(\alpha_{-} n_{1, y}+\alpha_{+} n_{2, y}\right)$,

where $\hat{\mathbf{n}}_{1}\left(\hat{\mathbf{n}}_{2}\right)$ is the unit vector in the direction of the nucleon (antinucleon) in the rest frame of $\Lambda$ $(\bar{\Lambda})$. The components of these vectors are expressed using a common coordinate system defined in Ref. [2]. The terms multiplied by $\alpha_{-} \alpha_{+}$represent the contribution from $\Lambda \bar{\Lambda}$ spin correlations, while the terms multiplied by $\alpha_{-}$and $\alpha_{+}$separately represent the contribution from the polarizations. If all three contributions in Eq. (1.1) are non-zero an unambiguous determination of the parameters $\alpha_{\psi}$ and $\Delta \Phi$ and the decay asymmetries $\alpha_{-}, \alpha_{+}$is possible.

The analysis is based on $1.31 \times 10^{9} \mathrm{~J} / \psi$ events collected with the BESIII detector. The $\Lambda$ hyperons are reconstructed using their $p \pi^{-}$decays and the $\bar{\Lambda}$ hyperons using their $\bar{p} \pi^{+}$or $\bar{n} \pi^{0}$ decays. The sizes of the final data samples are 420,593 and 47,009 events with an estimated background of $399 \pm 20$ and $66.0 \pm 8.2$ events for the $p \pi^{-} \bar{p} \pi^{+}$and $p \pi^{-} \bar{n} \pi^{0}$ final states, respectively. The background contribution is determined from Monte Carlo (MC) simulation including all known $J / \psi$ decays. For each event the full set of the kinematic variables $\boldsymbol{\xi}$ is reconstructed. The free parameters describing the angular distributions for the two data sets $-\alpha_{\psi}, \Delta \Phi, \alpha_{-}, \alpha_{+}$, and $\bar{\alpha}_{0}$ - are determined from a simultaneous unbinned maximum likelihood fit using angular distribution given by Eq. (1.1). A clear polarization, strongly dependent on the $\Lambda$ direction, $\cos \theta$, is observed for $\Lambda$ and $\bar{\Lambda}$. In Fig. 1, the moment $\mu(\cos \theta)=(m / N) \sum_{i}^{N_{k}}\left(n_{1, y}^{(i)}-n_{2, y}^{(i)}\right)$, related to the polarization, is calculated for $m=50$ bins in $\cos \theta . N$ is the total number of events in the data sample and $N_{k}$ is the number of events in $k$-th $\cos \theta$ bin. The expected angular dependence is $\mu(\cos \theta) \sim \sqrt{1-\alpha_{\psi}^{2}}\left(\alpha_{-}-\alpha_{+}\right) \sin \Delta \Phi \cos \theta \sin \theta$ for the acceptance corrected data (compare 

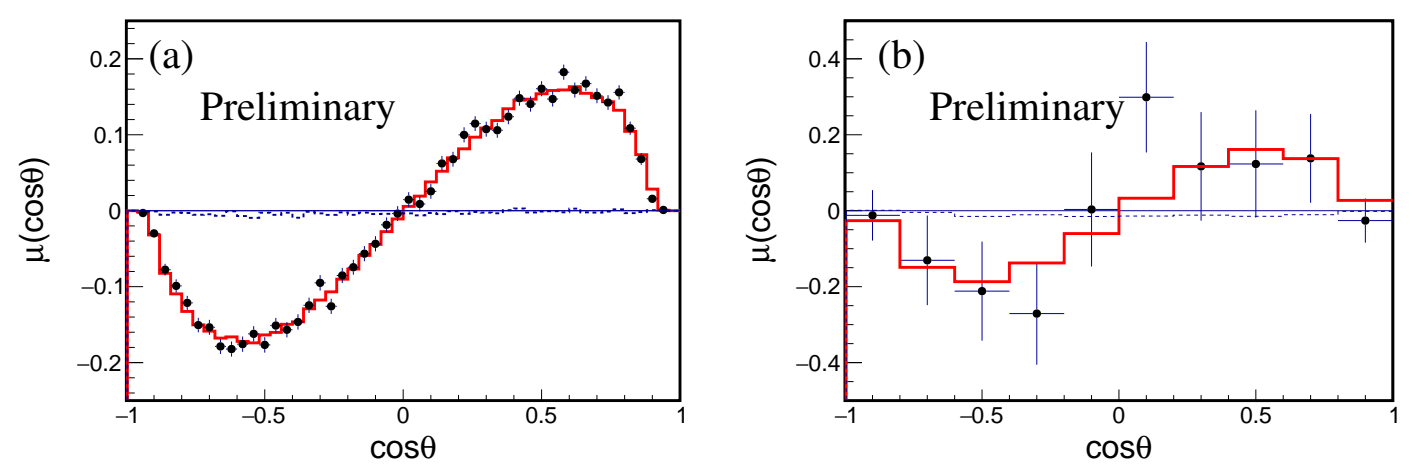

Figure 1: Moments $\mu(\cos \theta)$ as a function of $\cos \theta$ for $e^{+} e^{-} \rightarrow\left(\Lambda \rightarrow p \pi^{-}\right)\left(\bar{\Lambda} \rightarrow \bar{p} \pi^{+}\right)$data samples at (a) $\sqrt{s}=3.096 \mathrm{GeV}(J / \psi$ mass $)$ and (b) $\sqrt{s}=2.396 \mathrm{GeV}$. The points with error bars are the data, and the solid-line histograms are the global fit results. The dashed histograms show the no polarization scenario $(\mathscr{W}(\boldsymbol{\xi} ; 0,0,0,0) \equiv 1)$.

Table 1: Summary of the results: the $J / \psi \rightarrow \Lambda \bar{\Lambda}$ angular distribution parameter $\alpha_{\psi}$, the phase $\Delta \Phi$, the asymmetry parameters for the $\Lambda \rightarrow p \pi^{-}\left(\alpha_{-}\right), \bar{\Lambda} \rightarrow \bar{p} \pi^{+}\left(\alpha_{+}\right)$and $\bar{\Lambda} \rightarrow \bar{n} \pi^{0}\left(\bar{\alpha}_{0}\right)$ decays, the $C P$ asymmetry $A_{C P}$, and the ratio $\bar{\alpha}_{0} / \alpha_{+}$.

\begin{tabular}{lcc}
\hline \hline Parameters & This work & Previous results \\
\hline$\alpha_{\psi}$ & $0.461 \pm 0.006 \pm 0.007$ & $0.469 \pm 0.027[1]$ \\
$\Delta \Phi$ & $(42.4 \pm 0.6 \pm 0.5)^{\circ}$ & - \\
\hline$\alpha_{-}$ & $0.750 \pm 0.009 \pm 0.004$ & $0.642 \pm 0.013[8]$ \\
$\alpha_{+}$ & $-0.758 \pm 0.010 \pm 0.007$ & $-0.71 \pm 0.08 \quad[8]$ \\
$\bar{\alpha}_{0}$ & $-0.692 \pm 0.016 \pm 0.006$ & - \\
$A_{C P}$ & $-0.006 \pm 0.012 \pm 0.007$ & $0.006 \pm 0.021[8]$ \\
$\bar{\alpha}_{0} / \alpha_{+}$ & $0.913 \pm 0.028 \pm 0.012$ & - \\
\hline \hline
\end{tabular}

Eq. (1.1)). The phase between helicity flip and helicity conserving transitions is determined to be $\Delta \Phi=(42.4 \pm 0.6 \pm 0.5)^{\circ}$. This large value of the phase enables a simultaneous determination of the decay asymmetry parameters for $\Lambda \rightarrow p \pi^{-}, \bar{\Lambda} \rightarrow \bar{p} \pi^{+}$, and $\bar{\Lambda} \rightarrow \bar{n} \pi^{0}$ as given in Table 1. The value of $\alpha_{-}=0.750 \pm 0.009 \pm 0.004$ differs by more than five standard deviations from the world average value of $\alpha_{-}^{\mathrm{PDG}}=0.642 \pm 0.013$ established in 1978 [9]. However, in our opinion the uncertainty on $\alpha_{-}^{\mathrm{PDG}}$ should be larger since it does not include e.g. a systematic uncertainty of at least $5 \%$ in the two most precise results $[10,11]$. Our value implies that all published measurements on $\Lambda / \bar{\Lambda}$ polarization are $(17 \pm 3) \%$ too large.

\section{The $e^{+} e^{-} \rightarrow \Lambda \bar{\Lambda}$ process at the continuum ([12] and preliminary)}

In a run at $\sqrt{s}=2.396 \mathrm{GeV}$ with integrated luminosity of $66.9 \mathrm{pb}^{-1}$ a data sample of 555 $e^{+} e^{-} \rightarrow \Lambda \bar{\Lambda}\left(\Lambda \rightarrow p \pi^{-}\right.$and $\left.\bar{\Lambda} \rightarrow \bar{p} \pi^{+}\right)$candidate events was collected. The estimated background is $14 \pm 4$. This allows measurement of $\Lambda$ hyperon form factors, the first complete determination of time-like elastic form factors (EFFs) for any baryon. In the analysis the joint angular distribution given by Eq. (1.1) is used. The helicity non-flip and flip amplitudes are identified with 
the electric and magnetic form factors $G_{E}$ and $G_{M}$, respectively. The complete information about the form factors is represented by the Born cross section, the ratio $R=\left|G_{E} / G_{M}\right|$ and the phase $\Delta \Phi=\arg \left(G_{E} / G_{M}\right)$. Using the values for the decay asymmetry parameters $\alpha_{-}$and $\alpha_{+}$from the BESIII analysis described in Sec. 1 as the input, the obtained values are: $R=0.96 \pm 0.14 \pm 0.02$ and $\Delta \Phi=(37 \pm 12 \pm 6)^{\circ}$. The observed non-zero phase is a clear demonstration that the $\Lambda$ EFFs cannot be described by rational functions of the four-momentum transfer squared [5]. The Born cross section of the process is measured to be $119.0 \pm 5.3 \pm 5.1 \mathrm{pb}$.

In a sepatate analysis the Born cross section for the process $e^{+} e^{-} \rightarrow \Lambda \bar{\Lambda}$ was measured at $\sqrt{s}=2.2324 \mathrm{GeV}$ [12], i.e. $1.0 \mathrm{MeV}$ above threshold, to be $305 \pm 45_{-36}^{+66} \mathrm{pb}$. Such large value is unexpected since for neutral baryons the cross section is not enhanced by the Coulomb factor and should vanish at threshold.

\section{References}

[1] BESIII collaboration, M. Ablikim et al., Study of $J / \psi$ and $\psi(3686)$ decay to $\Lambda \bar{\Lambda}$ and $\Sigma^{0} \bar{\Sigma}^{0}$ final states, Phys. Rev. D95 (2017) 052003.

[2] BESIII collaboration, M. Ablikim et al., Polarization and Entanglement in Baryon-Antibaryon Pair Production in Electron-Positron Annihilation, arXiv:1808.08917.

[3] L. Kopke and N. Wermes, J/ $\psi$ Decays, Phys. Rept. 174 (1989) 67.

[4] N. Cabibbo and R. Gatto, Electron Positron Colliding Beam Experiments, Phys. Rev. 124 (1961) 1577.

[5] S.J. Brodsky, C.E. Carlson, J.R. Hiller and D.S. Hwang, Single spin polarization effects and the determination of time - like proton form-factors, Phys. Rev. D69 (2004) 054022.

[6] G. Fäldt and A. Kupsc, Hadronic structure functions in the $e^{+} e^{-} \rightarrow \bar{\Lambda} \Lambda$ reaction, Phys. Lett. B772 (2017) 16.

[7] T.D. Lee and C.N. Yang, General Partial Wave Analysis of the Decay of a Hyperon of Spin 1/2, Phys. Rev. 108 (1957) 1645.

[8] Particle Data Group collaboration, C. Patrignani et al., Review of Particle Physics, Chin. Phys. C40 (2016) 100001.

[9] Particle Data Group collaboration, C. Bricman et al., Review of Particle Properties. Particle Data Group, Phys. Lett. 75B (1978) 1.

[10] O.E. Overseth and R.F. Roth, Time Reversal Invariance in $\Lambda$ Decay, Phys. Rev. Lett. 19 (1967) 391.

[11] W.E. Cleland, G. Conforto, G.H. Eaton, H.J. Gerber, M. Reinharz, A. Gautschi et al., A measurement of the $\beta$-parameter in the charged nonleptonic decay of the $\Lambda$ hyperon, Nucl. Phys. B40 (1972) 221.

[12] BESIII collaboration, M. Ablikim et al., Observation of a cross-section enhancement near mass threshold in $e^{+} e^{-} \rightarrow \Lambda \bar{\Lambda}$, Phys. Rev. D97 (2018) 032013 [arXiv:1709.10236]. 